\title{
The young open cluster NGC 663 and its Be stars ${ }^{\star}$
}

\author{
A. Pigulski, G. Kopacki, and Z. Kołaczkowski \\ Wrocław University Observatory, Kopernika 11, 51-622 Wrocław, Poland \\ Received 13 December 2000 / Accepted 14 June 2001

\begin{abstract}
We present new $B V(R I)_{\mathrm{C}} \mathrm{H} \alpha$ photometry of the young open cluster NGC 663 . The $\mathrm{H} \alpha$ photometry is complete down to magnitude $R_{\mathrm{C}}=15.4$, corresponding to spectral type A5 for the cluster members. This allows detection of mild and strong $\mathrm{H} \alpha$ emission in all B-type stars in the cluster. In addition to the 22 Be stars known in the observed field of NGC 663, we discovered four new faint stars of this type. We find that Be stars in NGC 663 cover the whole range of the B spectral type. They are, however, most populous among stars with spectral types falling in the range between B0 and B3, where their fraction amounts to $31 \pm 8 \%$. Among B-type stars later than B3, Be stars are much less abundant: only 7 out of 101 observed stars, that is, $7 \pm 3 \%$, were detected. About $70 \%$ of the observed Be stars in NGC 663 show detectable variations of light. In the time interval covered by our observations, the ranges of the largest variations reach 0.4 mag in the $I_{\mathrm{C}}$ band. By means of the isochrone fitting, we derived the cluster distance of $2.1 \mathrm{kpc}$, age of 20-25 Myr, and the mean colour excess $E(R-I)_{\mathrm{C}}=$
\end{abstract} \\ $0.54 \mathrm{mag}$, with a $\sim 0.1 \mathrm{mag}$ scatter due to differential reddening.
}

Key words. stars: emission-line, Be - open clusters and associations: individual: NGC 663

\section{Introduction}

The differences in Be-star fractions in open clusters are still not well understood. Some investigators postulate age (Fabregat \& Torrejón 2000) and metallicity (Maeder et al. 1999) effects. In order to study this problem, the Be star fractions have to be known in many clusters and in the whole range of $\mathrm{B}$ spectral types. Unfortunately, the observed fractions of Be stars are very often systematically biased because the searches are not complete. This is mainly true in the case of spectroscopic searches which, for practical reasons, were usually confined to the brightest and most well-isolated stars in a cluster. As a consequence, Be star fraction for late B-type stars was often underestimated. The situation was improved when the era of CCD $\mathrm{H} \alpha$ photometry began. Although with this technique faint emission cannot be detected, it works well in crowded fields and needs much less telescope time than does spectroscopy. Even with a small-size telescope, late B-type stars in many open clusters can be easily reached, so that complete information on the Be star content can be obtained.

Detection of Be stars by means of the CCD photometry is usually carried out with a filter centred on $\mathrm{H} \alpha$ and

Send offprint requests to: A. Pigulski,

e-mail: pigulski@astro.uni.wroc.pl

* Tables 2 and 3 are only available in electronic form at the CDS via anonymous ftp to cdsarc.u-strasbg.fr (130.79.128.5) or via

http://cdsweb.u-strasbg.fr/cgi-bin/qcat?J/A+A/376/144 two other filters located in the Paschen continuum (see, e.g., Grebel 1997). It is even better when two filters of different width centred on $\mathrm{H} \alpha$ are used, because they may define a photometric index independent of the interstellar extinction (see, e.g., Goderya \& Schmidt 1994). Such a pair of $\mathrm{H} \alpha$ filters was used in our observations.

NGC 663 is a young galactic open cluster very rich in Be stars. According to Sanduleak (1979), it contains over twenty Be stars, that is, more than one third of all its early B-type stars. This is why this cluster was chosen as one of the first targets in our Be star survey among northern open clusters. Within this search, we have recently discovered that NGC 7419, another open cluster of similar age, contains over thirty Be stars (Pigulski \& Kopacki 2000). Our project is also complementary to the ongoing search for variables of type B in young open clusters (see Pigulski et al. 2000 and references therein).

\section{Observations}

All observations of NGC 663 were carried out at the Białków Observatory of the Wrocław University with a 60 -cm telescope equipped with a $576 \times 384$ pixel $\mathrm{CCD}$ camera and an autoguider. Since the camera field of view is a $6^{\prime} \times 4^{\prime}$ rectangle, 15 overlapping fields were covered in order to observe most of the cluster stars. The borders of the observed field, called hereafter the "P field", are shown with a solid line in Fig. 1, while a detailed map, showing all stars we measured, is given in Fig. 2. 


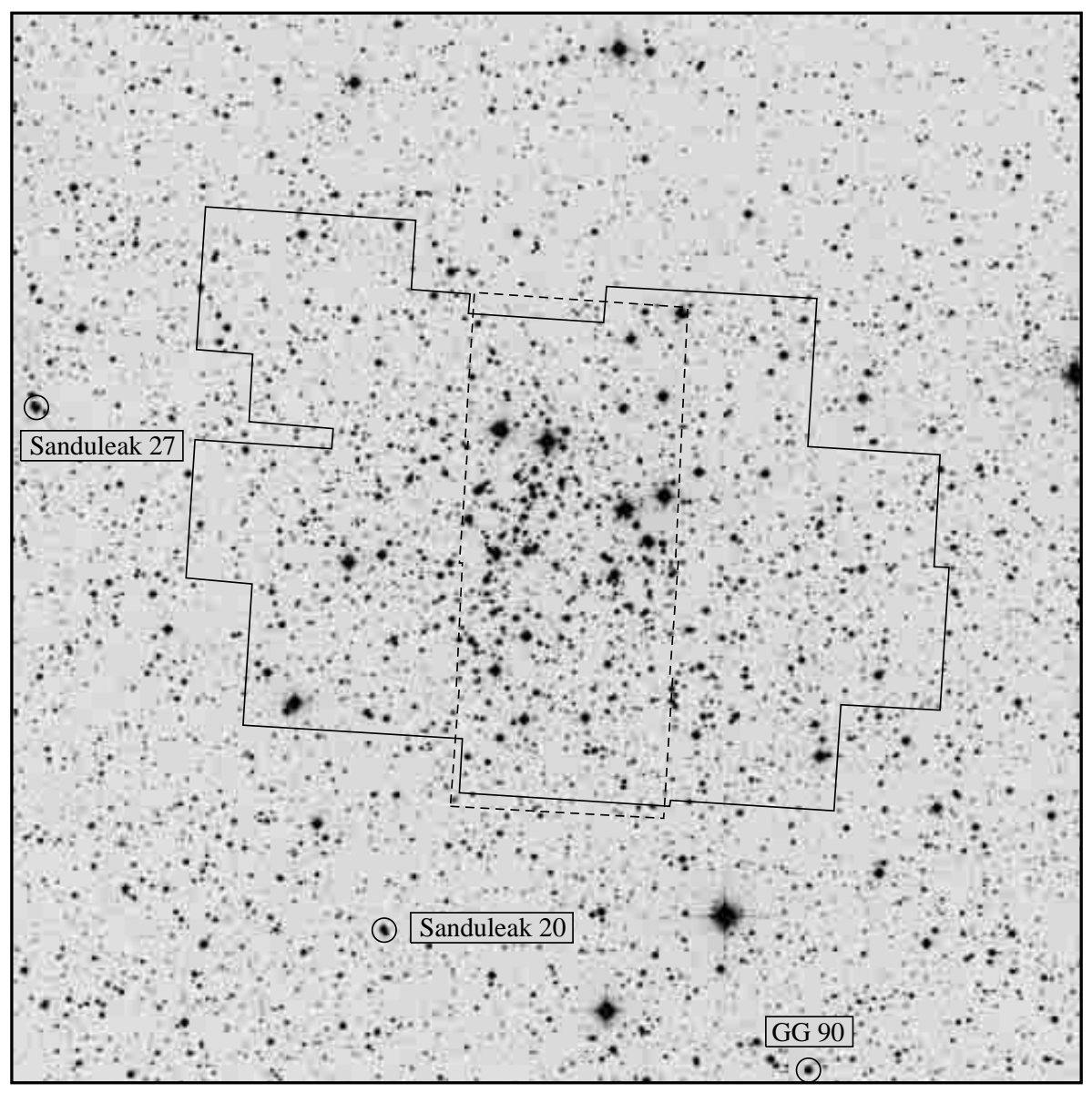

Fig. 1. A $30^{\prime} \times 30^{\prime}$ fragment of the POSS I plate centred on NGC 663. Solid lines show the border of the area covered by our $B V(R I)_{\mathrm{CH}} \alpha$ observations (P field). The dashed lines delimit the region searched for variability by Pigulski et al. (2001); we call it the $\mathrm{V}$ field. Three Be stars from outside the $\mathrm{P}$ field are encircled and labeled. North is up, east to the left.

The observations in each field consisted of a series of frames taken through filters reproducing the $B V(R I)_{\mathrm{C}}$ bands of the Johnson-Cousins (Johnson \& Morgan 1953; Cousins 1976) system and a single frame taken through the narrow $\mathrm{H} \alpha$ filter. The latter was usually sandwiched between two frames taken through the wide $\mathrm{H} \alpha$ filter. The observations were carried out during 10 nights between March 7, 1997 and September 1, 1998. Typical exposure times were the following: $2000 \mathrm{~s}$ for narrow $\mathrm{H} \alpha, 600 \mathrm{~s}$ for wide $\mathrm{H} \alpha$ and $B$, and less than $200 \mathrm{~s}$ for the remaining filters.

NGC 663 was also observed in 1999 in the abovementioned program of searching for variable B-type stars in the northern open clusters. During these observations, however, only four slightly overlapping fields were observed in $V$ and $I_{\mathrm{C}}$ bands. The area covered during this search is shown in Fig. 1 with dashed lines and will be called hereafter the "V field". The results of the variability survey in the $\mathrm{V}$ field will be published separately (Pigulski et al. 2001). In this paper we present only that part of these results which refer to the variability of Be stars (see Sect. 6).

The CCD frames were calibrated in a standard way (see, e.g., Jerzykiewicz et al. 1997) and then reduced using the point-spread-function fitting in the Daophot II package of Stetson (1987).

\section{3. $B V(R I)_{\mathrm{C}}$ photometry}

\subsection{Transformations}

The $B V$ observations of NGC 663 were transformed using mainly the photoelectric observations of Hoag et al. (1961). The photographic observations of the same authors were used only in transformation of $V$, because their photographic $B$ photometry is systematically biased (see Fig. 3). The transformation equations for $B$ and $V$ are the following:

$$
\begin{aligned}
& B=b-0.002(63) \times(b-v)+12.801(6), \\
& V=v+0.070(29) \times(v-i)+12.198(8),
\end{aligned}
$$

where the numbers in parentheses denote the rms errors of the coefficients with the leading zeroes omitted. The lower-case letters are instrumental differential magnitudes calculated with respect to a set of relatively bright constant stars. Standard deviations of residuals from Eqs. (1) and (2) were 0.024 and $0.056 \mathrm{mag}$, respectively. 


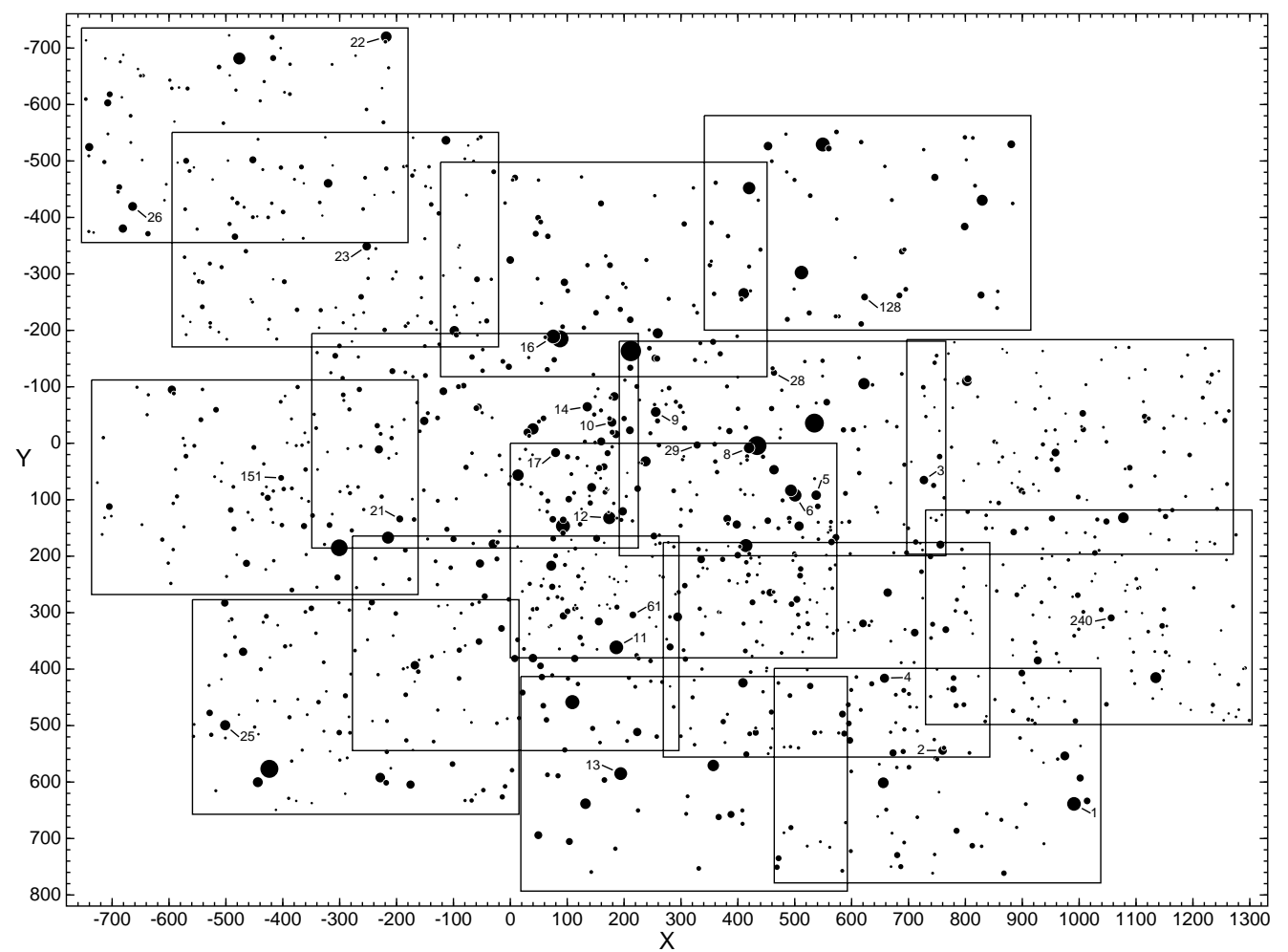

Fig. 2. Schematic view of our fifteen $6^{\prime} \times 4^{\prime}$ fields in NGC 663, covering the $\mathrm{P}$ field. The diameters of the circles correspond to the stars' images as seen through the $R_{\mathrm{C}}$ filter. All emission-line objects listed in Table 1 are labeled with their numbers from Sanduleak (smaller than 30) or Wallenquist (1929, larger than 30) list. North is approximately up, east to the left.

Since till now no $(R I)_{\mathrm{C}}$ photometry was published for NGC 663, in order to transform our observations in these bands to the standard system, we observed NGC 663 on three photometric nights in September and October 2000 together with another open cluster, NGC 7790. We have chosen NGC 7790 instead of the usually observed Landolt (1992) fields for two reasons: (i) this cluster is much closer to NGC 663 than the Landolt (1992) standards, (ii) $(R I)_{\mathrm{C}}$ standard photometry of NGC 7790 has been recently published by Stetson (2000).

Using the photometry of NGC 7790 provided by Stetson (2000), we derived the following transformation equations:

$$
\begin{aligned}
& R_{\mathrm{C}}=r+0.031(24) \times(r-i)+11.909(4), \\
& I_{\mathrm{C}}=i+0.002(25) \times(r-i)+11.512(4),
\end{aligned}
$$

where the designations are similar to those in Eqs. (1) and (2), and the standard deviations of residuals from Eqs. (3) and (4) are equal to 0.011 and $0.012 \mathrm{mag}$, respectively. The resulting standard $B V(R I)_{\mathrm{C}}$ photometry of over 1200 stars is given in Table 2, available in the electronic form only, via anonymous ftp to CDS, Strasbourg (130.79.128.5). In Fig. 7 , the $R_{\mathrm{C}}$ vs. $(R-I)_{\mathrm{C}} \mathrm{CM}$ diagram is shown.

The photometry we provide in Table 2 is not homogeneous in the sense that the central regions were observed more frequently than those situated at the borders of the $\mathrm{P}$ field. In consequence, stars of the same magnitude, but from different regions, may have photometry which differs in accuracy by a factor of up to 5. For this reason, the photometric errors are also given in Table 2.

\subsection{Comparison with previous work}

Having transformed our $B V$ photometry to the standard system, we compared it with the following previous photometric studies: CCD measurements of Phelps \& Janes (1994), photoelectric photometry of Hoag et al. (1961), photographic data of the same authors as well as those of McCuskey \& Houk (1964), Moffat \& Vogt (1974), and van den Bergh \& de Roux (1978). Results of this comparison are shown in Fig. 3.

Photoelectric measurements of Hoag et al. (1961) were the primary source of standard stars used in our transformations. Hence the scatter in panel A of Fig. 3 is small. Out of four photographic photometries, the best agreement and the smallest scatter are shown by the photometry of van den Bergh \& de Roux (1978). The differences between our photometry and the photographic photometries of Moffat \& Vogt (1974) and McCuskey \& Houk (1964) have larger scatter, while that of Hoag et al. (1961), especially the $B$ photometry, exhibits a clear systematic, magnitude-dependent effect.

Surprisingly, the CCD photometry of Phelps \& Janes (1994) also differs systematically from our measurements. While for stars with magnitudes in the range between 12 

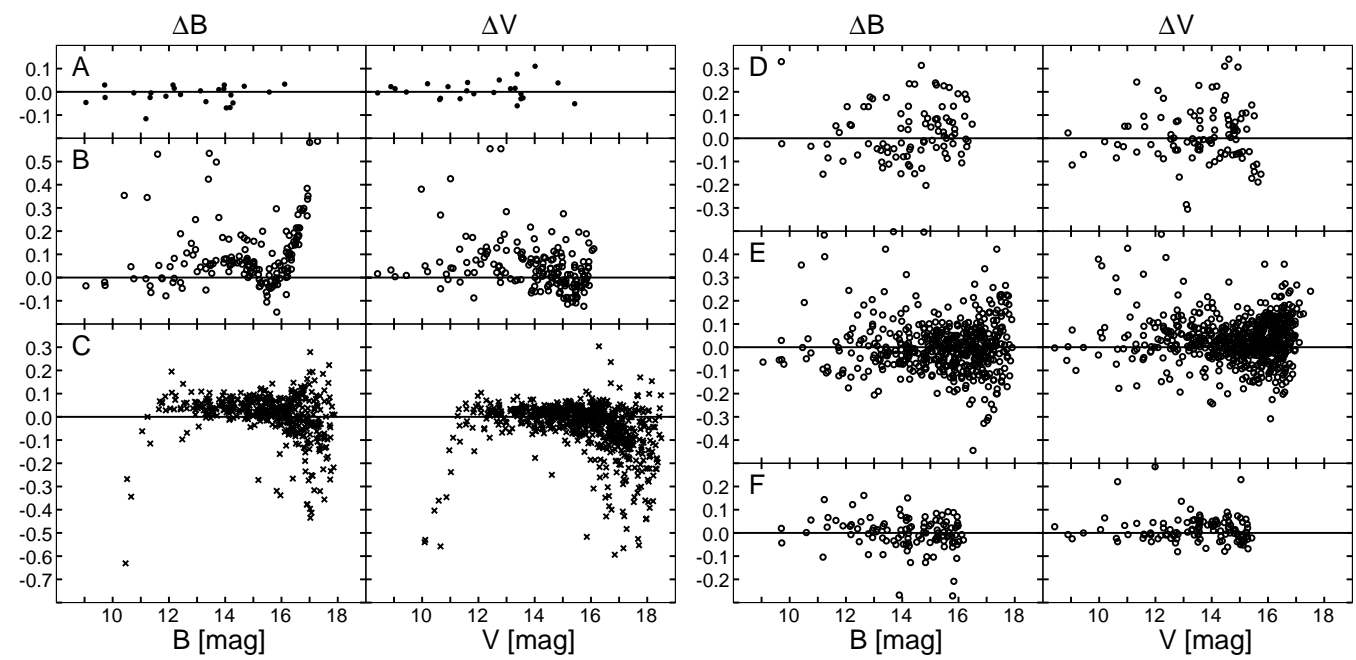

Fig. 3. Comparison of $B V$ photometries. All differences, $\Delta B$ and $\Delta V$, are in the sense our minus the other. The other photometries are designated by the following letters in the left upper corners of panels: a) Hoag et al. (1961), photoelectric; b) Hoag et al. (1961), photographic; c) Phelps \& Janes (1994); d) McCuskey \& Houk (1964); e) Moffat \& Vogt (1974), f) van den Bergh $\&$ de Roux (1978). The photoelectric data are shown with dots, photographic data, with open circles, and CCD data, with crosses.

and 16 in $B$ and $V$ the agreement is quite good (apart from a $0.06 \mathrm{mag}$ shift in $B$ ), for stars fainter than $16 \mathrm{mag}$, the photometry of Phelps \& Janes (1994) is systematically fainter and shows a large scatter. In addition, stars brighter than $\sim 12 \mathrm{mag}$ are also systematically fainter in their photometry. The same effect can be seen in both $B$ and $V$, reaching $0.5 \mathrm{mag}$ for stars of the 10th magnitude.

\section{Known Be stars}

The field of NGC 663 is one of the most populous as far as Be stars are concerned. In the following discussion of Be stars in this open cluster, we shall confine ourselves to the $30^{\prime} \times 30^{\prime}$ area shown in Fig. 1 .

The first Be star discovered in this area was MWC $428=\mathrm{BD}+60^{\circ} 325$ (Merrill \& Burwell 1943). A few years later, the next four Be stars: MWC $698=$ $\mathrm{BD}+60^{\circ} 332 \mathrm{~A}, \quad \mathrm{MWC} 700=\mathrm{BD}+60^{\circ} 340, \quad \mathrm{AS} 42=$ $\mathrm{BD}+60^{\circ} 341$, and $\mathrm{AS} 43=\mathrm{BD}+60^{\circ} 343 \mathrm{~B}$ were found by the same authors (Merrill \& Burwell 1949, 1950). Since subsequent observations did not detect emission in MWC 700 (known also as Sanduleak 11), its Be nature is not certain. As was pointed out by Sanduleak (1979), it could happen that the BD number was incorrectly assigned by Merrill \& Burwell (1949) and the star is identical with AS 42.

González \& González (1954) were the first to show that the cluster contains a large number of Be stars. They found 13 new stars of this type in the area under investigation. Subsequent studies (Dolidze 1975; Schild \& Romanishin 1976; Coyne et al. 1978; MacConnell \& Coyne 1983; Sanduleak 1990) added seven more stars, increasing the number of certain Be stars in NGC 663 to 24. These stars are listed in the compilation of Kohoutek \& Wehmeyer (1997).
Sanduleak (1979) has provided a list of 27 Be stars in NGC663, extending it by two objects in a subsequent paper (Sanduleak 1990). Since the numbers from Sanduleak's lists are widely used in the literature, we shall also refer to these stars preceding the number with "Sanduleak". We now know, however, that not all Sanduleak's stars are still considered to be Be stars. Sanduleak 7, 18, and 19, announced to be Be stars by Coyne et al. (1978), appeared to be non-emission stars after revision (MacConnell et al. 1983). In addition, two other stars, Sanduleak 15 and 24, were found to have doubtful or very weak emission (Schild \& Romanishin 1976; Voight 1965, respectively). Later observations (Sanduleak 1979, 1990; Torrejón et al. 1997; this paper) did not reveal emission in these stars either. Thus, only 23 stars with Sanduleak's numbers should be considered as certain Be stars and one, Sanduleak 11, as a probable Be star. We list these stars in Table 1. This table includes also five other emission-line stars: four new Be stars we discovered in the P field and star GG $90=$ D $01+030$ (see caption of Table 1 for designations) which probably is not a member, but is situated in the field shown in Fig. 1.

\section{5. $\mathrm{H} \alpha$ photometry}

The $\mathrm{H} \alpha$ photometry we made was used to find stars showing $\mathrm{H} \alpha$ emission. This was done by means of an $\alpha$ index, defined as a difference between the magnitudes of a star through a narrow and a wide filter. Our filters and, consequently, the $\alpha$ index resembles those used by others, namely Abt \& Golson (1966), Tebbe (1969), Feinstein (1974), Dachs \& Schmidt-Kaler (1975), Claría \& Escosteguy (1981), Strauss \& Ducati (1981), and Goderya \& Schmidt (1994). We made, however, no attempt to transform our $\alpha$ to that defined by others; it was left in 
Table 1. List of Be stars in the $30^{\prime} \times 30^{\prime}$ field around NGC 663. The columns are: (1), Sanduleak (1979, 1990) number, (2), Wallenquist (1929) number, (3), Kohoutek \& Wehmeyer (1997) number, (4) and (5), $X$ and $Y$ coordinate in Fig. 2, (6), right ascension (epoch 2000.0, in hours, minutes, and seconds), (7), declination (epoch 2000.0, in degrees, arcmin and arcsec), (8), $R_{\mathrm{C}}$ magnitude (in mag), (9), $\alpha$ index (in mag), (10), other designations, "MWC" and "AS" precede numbers taken from papers of Merrill \& Burwell (1943, 1949, 1950), "GG", from González \& González (1954), "D", from Dolidze (1975), "VES", from Coyne et al. (1978). Stars are listed in order of increasing right ascension.

\begin{tabular}{|c|c|c|c|c|c|c|c|c|c|}
\hline$S$ & $\mathrm{~W}$ & $H B H$ & $X$ & $Y$ & Right Asc. & Decl. & $R_{\mathrm{C}}$ & $\alpha$ & Other designations \\
\hline (1) & $(2)$ & (3) & (4) & (5) & (6) & (7) & (8) & (9) & (10) \\
\hline - & 240 & - & 1056.6 & 309.1 & 14511.65 & +611022.4 & 12.972 & 2.034 & new Be star \\
\hline 1 & 243 & $6210-32$ & 990.8 & 638.9 & 14518.02 & +610655.9 & 9.902 & 1.463 & $\mathrm{BD}+60^{\circ} 325, \mathrm{MWC} 428, \mathrm{D} 01+028$ \\
\hline - & 417 & - & & - & 14518.31 & +605809.2 & & & GG 90, D $01+030$ \\
\hline 2 & 210 & $6210-54$ & 759.9 & 544.1 & 14537.77 & +610759.2 & 12.054 & 1.330 & GG 93, VES 610, D 01+031 \\
\hline 3 & 130 & - & 727.3 & 65.1 & 14539.63 & +611259.6 & 12.094 & 1.388 & GG 94, VES 611 \\
\hline 4 & 170 & - & 657.6 & 415.8 & 14546.35 & +610920.9 & 11.771 & 1.977 & GG 95 \\
\hline - & 128 & - & 622.8 & -259.1 & 14547.85 & +611623.6 & 13.122 & 1.949 & new Be star \\
\hline 5 & 93 & $6210-56$ & 537.6 & 91.8 & 14556.07 & +611245.3 & 11.585 & 1.444 & GG 97, VES 613 \\
\hline 6 & 92 & $6210-57$ & 500.6 & 92.2 & 14559.27 & +611245.6 & 10.230 & 1.982 & $\mathrm{BD}+60^{\circ} 332 \mathrm{~A}, \mathrm{MWC} 698$, VES 614 \\
\hline 28 & 84 & $6210-34$ & 463.4 & -125.1 & 14602.03 & +611502.1 & 13.535 & 1.333 & \\
\hline 8 & 53 & $6210-15$ & 419.8 & 8.5 & 14606.09 & +611339.1 & 11.115 & 1.388 & $\mathrm{BD}+60^{\circ} 333 \mathrm{~B}, \mathrm{VES} 616$ \\
\hline 29 & 51 & $6210-73$ & 328.6 & 3.3 & 14613.99 & +611343.8 & 12.846 & 1.820 & \\
\hline 9 & 10 & $6210-31$ & 255.8 & -55.6 & 14620.19 & +611421.6 & 11.442 & 1.741 & GG 98, VES 617 \\
\hline 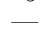 & 61 & - & 215.7 & 304.1 & 14624.37 & +611037.3 & 13.159 & 1.610 & new Be star \\
\hline 13 & 141 & $6210-13$ & 194.0 & 585.1 & 14626.80 & +610741.8 & 10.197 & 1.341 & $\mathrm{BD}+60^{\circ} 341, \mathrm{AS} 42, \operatorname{VES} 619, \mathrm{D} 01+033$ \\
\hline 10 & 8 & - & 178.8 & -37.1 & 14626.87 & +611411.2 & 11.905 & 1.999 & GG 99 \\
\hline 11 & 107 & - & 186.3 & 361.3 & 14627.02 & +611002.0 & 9.892 & 2.130 & $\mathrm{BD}+60^{\circ} 340, \mathrm{MWC} 700$ \\
\hline 12 & 21 & - & 174.1 & 132.2 & 14627.64 & +611225.5 & 10.341 & 1.725 & \\
\hline 14 & 6 & $6210-36$ & 135.1 & -64.9 & 14630.60 & +611429.3 & 11.656 & 1.446 & VES 620 \\
\hline 16 & 39 & - & 75.7 & -189.1 & 14635.49 & +611548.0 & 9.851 & 1.680 & $\mathrm{BD}+60^{\circ} 343 \mathrm{~B}, \mathrm{AS} 43, \mathrm{GG} 102$ \\
\hline 17 & 2 & $6210-14$ & 79.9 & 16.6 & 14635.57 & +61 1339.2 & 11.857 & 1.374 & GG 101 \\
\hline 20 & 288 & $6210-59$ & & & 14657.53 & +610140.4 & - & - & GG 103 \\
\hline 21 & 121 & $6210-51$ & -194.5 & 134.2 & 14659.55 & +611229.8 & 13.012 & 1.565 & VES 624 \\
\hline 22 & 194 & $6210-50$ & -218.3 & -720.0 & 14700.17 & +612123.7 & 11.039 & 1.837 & GG 104, VES 625 \\
\hline 23 & 124 & $6210-33$ & -252.3 & -349.0 & 14703.70 & $+61 \quad 1732.2$ & 12.072 & 2.050 & D $01-034$ \\
\hline & 151 & & -402.9 & 61.2 & 14717.46 & +611318.2 & 13.870 & 1.859 & new Be star \\
\hline 25 & 222 & $6210-35$ & -501.4 & 499.3 & 14726.72 & +610844.8 & 11.264 & 1.963 & GG 108, VES 628 \\
\hline 26 & 224 & $6210-25$ & -663.8 & -419.7 & 14739.34 & +611820.7 & 11.908 & 1.426 & GG 109, VES 630, D 01+036 \\
\hline 27 & 297 & $6210-26$ & - & - & 14823.04 & +611553.2 & - & - & GG 110 \\
\hline
\end{tabular}

the instrumental system. In Fig. 4 we show the $\alpha$ index for 442 stars with $R_{\mathrm{C}}<15.4 \mathrm{mag}$, the limiting magnitude of our $\mathrm{H} \alpha$ photometry. The same stars are shown in Fig. 5, where the $\alpha$ index is plotted as a function of $(R-I)_{\mathrm{C}}$.

Out of the 25 known Be stars in the area shown in Fig. 1, 22 fall within the $\mathrm{P}$ field, and 14 within the $\mathrm{V}$ field. As can be seen in Fig. 4, except for doubtful Be star Sanduleak 11, all these 22 known Be stars indeed show emission, that is, have $\alpha$ placing them above the locus of cluster non-emission stars, shown with a continuous line. The line was obtained from the spectra given by Danks \& Dennefeld (1994) which were combined with the transmission curves of the $\mathrm{H} \alpha$ filters provided by the manufacturer. Except for known Be stars, some other stars are found above this line as well. Because some foreground late-type stars can also have weak emission, we need to separate them from the Be stars first. For this reason, we made a rough selection of non-members with the use of the cluster CM diagram (see Sect. 7). Stars selected as non-members are plotted with crosses in Figs. 4 and 5. Obviously, with this kind of selection, we cannot indicate all non-members which contaminate the cluster main sequence. Many objects with $R_{\mathrm{C}}>14 \mathrm{mag}$ in Fig. 4 situated clearly above the continuous line are certainly such unrecognized nonmembers.

Using Fig. 4 we can conclude that four stars, namely W 61, 128, 151, and 240 (we precede star numbers taken from Wallenquist (1929) with "W"), may be classified as new Be stars. A star was selected as a new Be star if: (i) the difference between the observed $\alpha$ and the value taken for a star of the same magnitude showing no emission (continuous line in Fig. 4) was negative and its absolute value was larger than $4 \sigma_{\alpha}$, where $\sigma_{\alpha}$ is the rms error of $\alpha$, and (ii) the star was not selected as a non-member.

All four new Be stars are among the faintest Be stars in NGC 663, implying that the previous Be star surveys were rather complete for the brightest cluster stars. It can be also seen in Fig. 4 that Be stars with strong and intermediate $\mathrm{H} \alpha$ emission occur in the whole range of the B spectral type. Most of them, however, occupy the range between B0 and B3. Maeder et al. (1999) compared the fractions of Be stars in clusters of an age comparable to that of NGC 663. In the widest interval considered by these authors, $-5<M_{V}<-1.4 \mathrm{mag}$, they found that NGC 663 contained $34 \pm 11 \%$ of Be stars. The abovegiven interval of $M_{V}$ corresponds to $8.7<R_{\mathrm{C}}<12.2 \mathrm{mag}$. There are 59 stars we observed in this interval of $R_{\mathrm{C}}$, including 18 Be stars. This gives the revised fraction of $\mathrm{Be}$ stars in NGC 663 equal to $31 \pm 8 \%$, with the error calculated assuming Poisson statistics.

Among stars fainter than $R_{\mathrm{C}}=12.2 \mathrm{mag}$, we find 7 Be stars, including all four discovered in our search. Comparing this number with the number of all B-type stars later than B3 (101), we obtain the fraction of $7 \pm 3 \%$. 


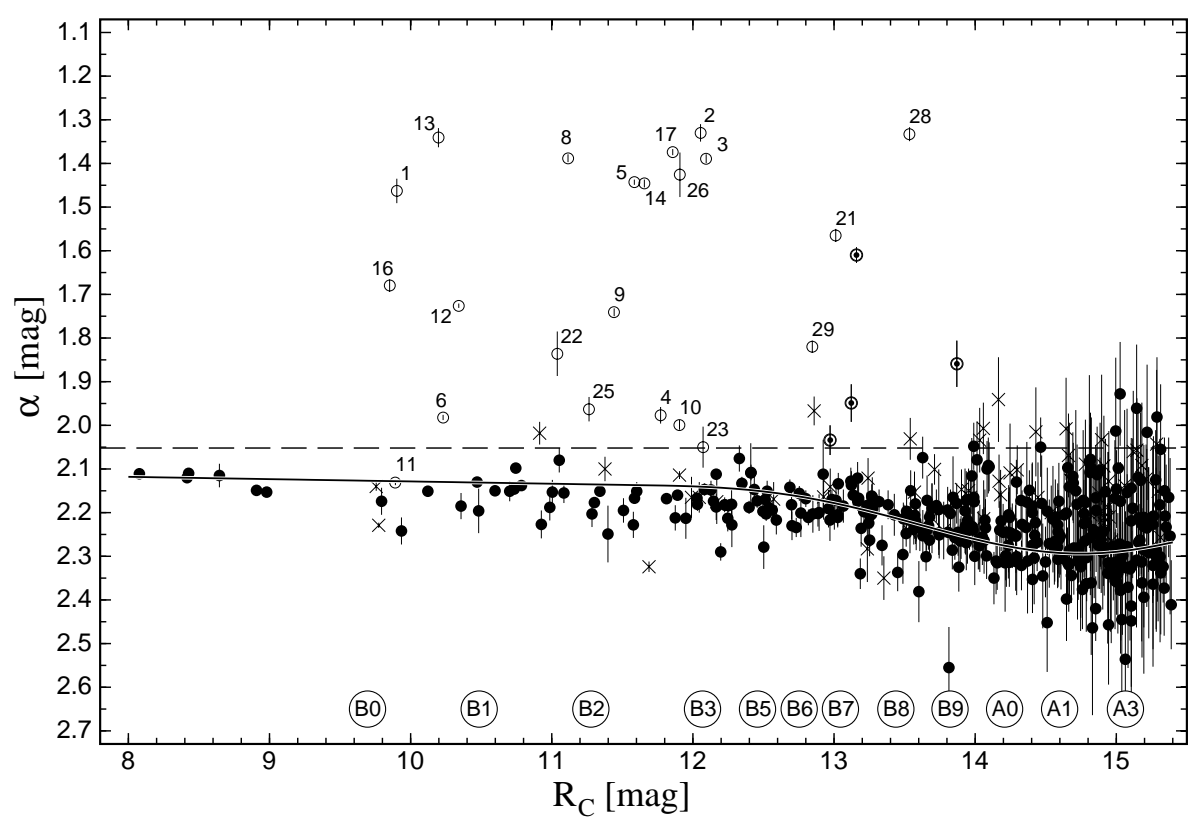

Fig. 4. The $\alpha$ index for the 442 stars brighter than $R_{\mathrm{C}}=15.4 \mathrm{mag}$, plotted as a function of $R_{\mathrm{C}}$. The symbols denote: crosses, non-members; open circles, known cluster Be stars; encircled dots, newly discovered Be stars; filled circles, remaining stars. Known Be stars are labeled with their Sanduleak $(1979,1990)$ numbers. The dashed line corresponds to the expected value of $\alpha$ for zero total equivalent width of the $\mathrm{H} \alpha$ line. The continuous line is an average relation for main-sequence non-emission $\mathrm{B}$ and early A-type members. At the bottom, the spectral types for the cluster main sequence stars are given.

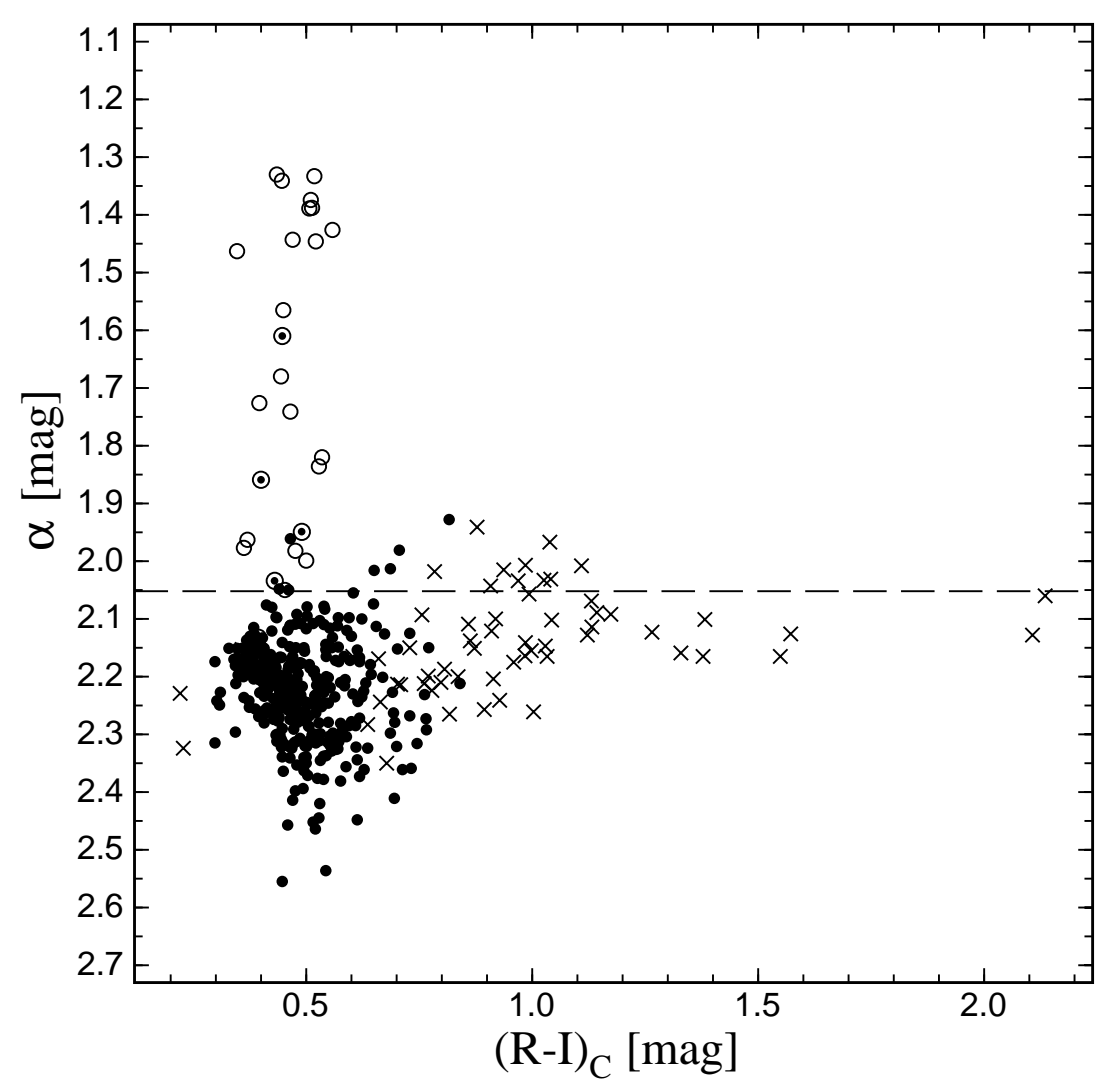

Fig. 5. The $\alpha$ index plotted versus the $(R-I)_{\mathrm{C}}$ colour. The symbols are the same as in Fig. 4 . For clarity, error bars and labels were omitted. 


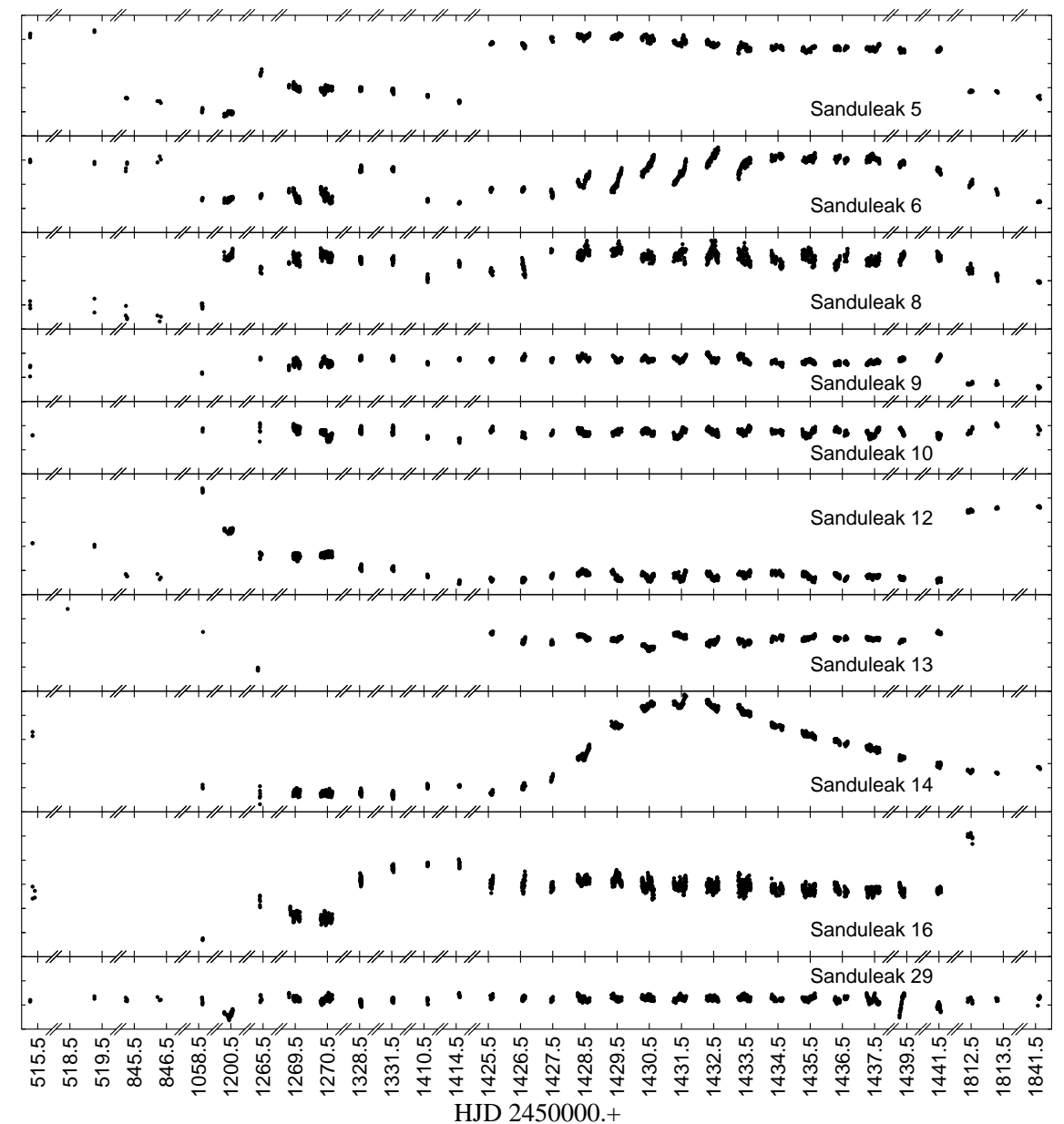

Fig. 6. The $I_{\mathrm{C}}$ light-curves of 10 Be stars from the V field of NGC 663. The ordinate ticks are separated by 0.1 mag. Note that the time axis is not continuous.

For 13 Be stars, simultaneous (in the sense that a narrow $\mathrm{H} \alpha$ frame was sandwiched between two wide $\mathrm{H} \alpha$ ones) $\mathrm{H} \alpha$ photometry was made in one epoch only. For the remaining 13 Be stars, such observations were made on two different epochs allowing detection of possible changes in the emission strength. The largest change of $\alpha$ we detected was $0.045 \mathrm{mag}$ for Sanduleak 3 and two epochs separated by about 550 days. The epochs of $\mathrm{H} \alpha$ observations and corresponding $\alpha$ values are given in Table 3, available in electronic form only.

The $\alpha$ indices given in Table 1 were calculated using all frames, including some additional wide $\mathrm{H} \alpha$ frames made on epochs other than those given in Table 3. For this reason the $\alpha$ indices given in Table 1 (average values) are not exactly the same as those presented in Table 3 (epoch photometry).

\section{Photometric variability of Be stars}

As we mentioned above, 14 Be stars from Table 1 are situated in the $\mathrm{V}$ field, the target of the variability survey. Out of these 14 stars, 10 were found to be variable (Pigulski et al. 2001) and only four (Sanduleak 11, 17, 28, and the newly discovered Be star, W 128) show no light variation above the detection limit. Nine variable Be stars show aperiodic or quasiperiodic variatons with ranges up to $0.4 \mathrm{mag}$ in $I_{\mathrm{C}}$. Only Sanduleak 10 is a periodic variable of $\lambda$ Eridani type with a period of $0.67298 \mathrm{~d}$ (Pigulski et al. 2001). Two drops of brightness seen in the light curve of Sanduleak 29 on HJD 2451200 and 2451439 could be eclipses. A large percentage of variables among Be stars is not unusual, because virtually all these stars exhibit some degree of photometric variability on different time-scales. The light curves of all variable Be stars situated in the V field are shown in Fig. 6.

Although observed on only two or three nights, some of the Be stars from outside the $\mathrm{V}$ field can also be classified as variables. These are: Sanduleak $1\left(\Delta I_{\mathrm{C}} \approx 0.39 \mathrm{mag}\right)$, $2\left(\Delta I_{\mathrm{C}} \approx 0.09 \mathrm{mag}\right), 3\left(\Delta I_{\mathrm{C}} \approx 0.10 \mathrm{mag}\right), 4\left(\Delta I_{\mathrm{C}} \approx\right.$ $0.10 \mathrm{mag})$, and $23\left(\Delta I_{\mathrm{C}} \approx 0.05 \mathrm{mag}\right)$. The remaining Be stars, that is, Sanduleak 11, 21, 22, 25, 26, W 128, 151, and 240 , show no clear evidence of variability exceeding the photometric errors of our photometry.

In order to plot an average position of the variable Be star in the CM diagram (Fig. 7), one should use observations made simultaneously in all bands. Such observations were chosen for averaging, but the epochs of the averaged magnitudes are not the same for all Be 


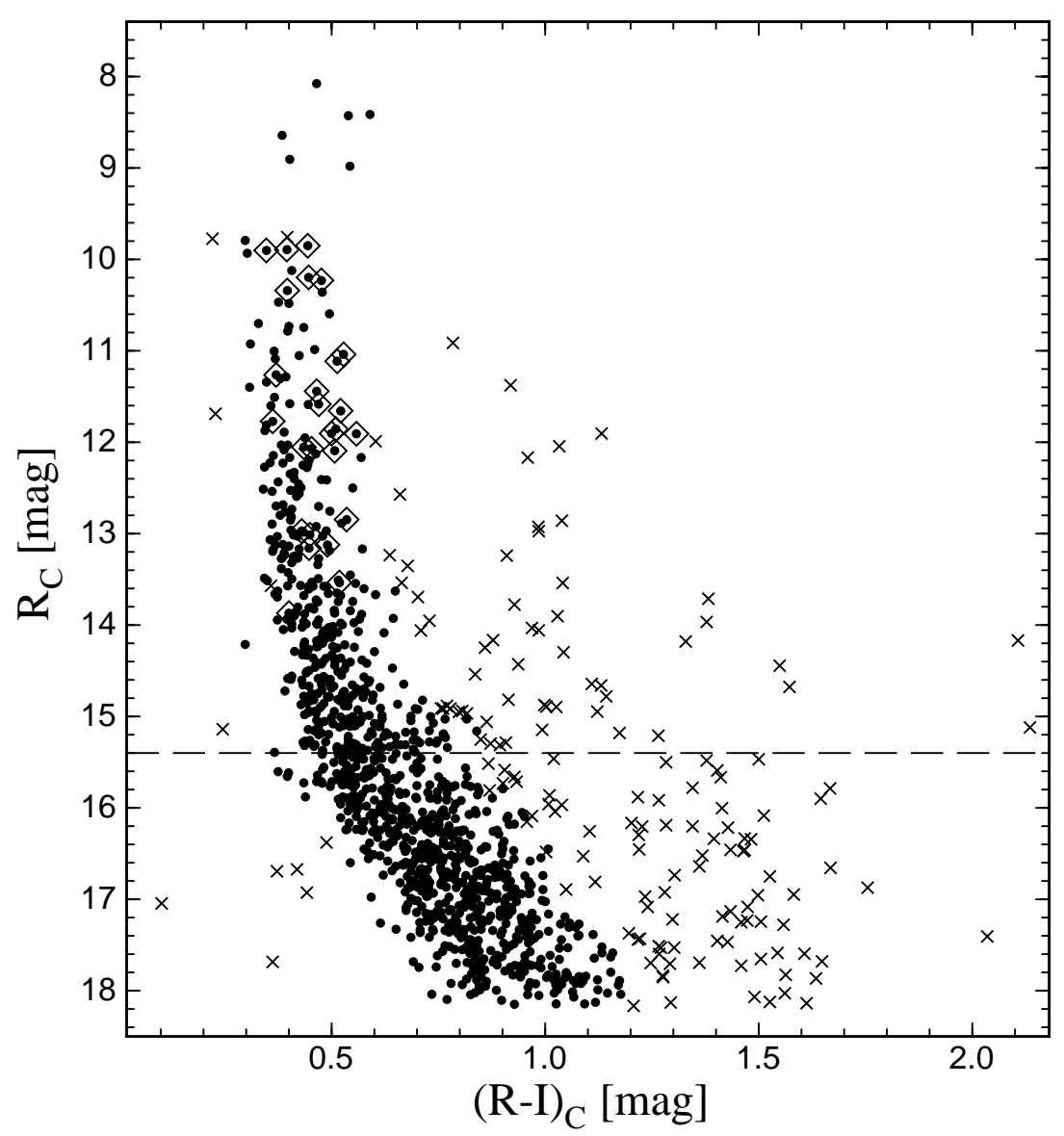

Fig. 7. CM diagram for NGC 663. Crosses denote stars identified as non-members (see Sect. 7). Diamonds are used to indicate the emission-line objects. The dashed line shows the limit of the $\mathrm{H} \alpha$ photometry.

variables (different fields were observed on different nights). These average epochs of the $B V(R I)_{\mathrm{C}}$ photometry are the following: HJD 2450515.3 for Sanduleak 9, 14, and 16; HJD 2450518.4 for Sanduleak 13; HJD 2450813.3 for Sanduleak 1, 2, and 4; HJD 2450845.3 for Sanduleak 23; HJD 2450846.3 for Sanduleak 5, 6, 8, 12, and 29; and HJD 2451 058.6 for Sanduleak 3. Observations of non-variable Be stars and Sanduleak 10 ( $\lambda$ Eridani-type variable) were averaged using all available photometry.

\section{The CM diagram and identification of non-members}

The members of NGC 663 form a well-defined main sequence in the CM diagram (Fig. 7). The main sequence is slightly widened by a differential reddening. Some nonmembers can be seen redward of the cluster main sequence. Non-members also contaminate the lower part of the main sequence, because with the mean reddening of NGC 663 (0.8 mag in terms of the $E(B-V)$ colour excess according to Phelps \& Janes 1994), foreground late-type dwarfs and the reddened cluster B- and A-type stars coincide in the CM diagram.

Since the late-type non-members also affect Fig. 4, we would like to distinguish them from the cluster members.
Although not unambiguous, the only selection we can practically perform is the selection that uses two-colour photometry. For instance, the non-members can be roughly selected by a dereddening procedure which takes into account the information on the spatial distribution of stars. Assuming that there is no reddening within the cluster and adopting a certain value of the spatial scale of reddening variation, characterized by a parameter $\varepsilon$ (see below), a reddening map can be derived and then used to correct the colours for differential reddening and magnitudes for differential extinction.

We made an attempt to calculate the variable part of the reddening with the procedure applied by Pigulski \& Kołaczkowski (1998) to the Cygnus OB2 association. We refer the reader to that paper for details of the calculations. Unlike that case, however, for NGC 663 we did not assume a priori the shape of the unreddened main sequence. Instead, the sequence was fitted by a third degree polynomial. The procedure was done iteratively in the following steps: (i) fitting the cluster main-sequence with a polynomial, (ii) calculating the differences between the observed position of a star and the intersection of the reddening line passing through that point with the main sequence (the difference was measured along the reddening line), (iii) calculating the reddening map with the use 


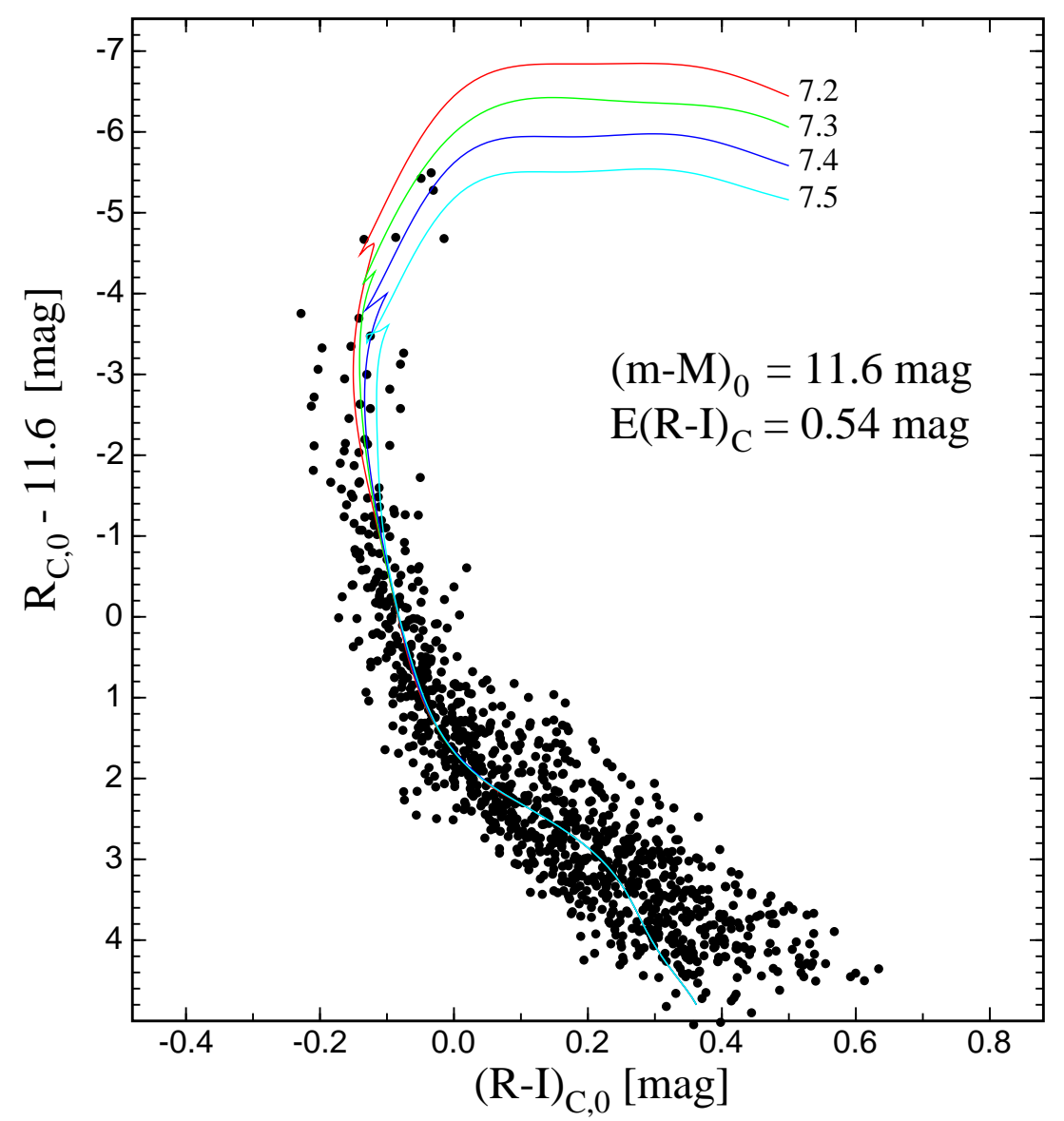

Fig. 8. Dereddened $R_{\mathrm{C}, 0}$ vs. $(R-I)_{\mathrm{C}, 0} \mathrm{CM}$ diagram for NGC 663 shifted in magnitude by the value of the distance modulus, $11.6 \mathrm{mag}$. Be stars and stars identified as non-members in Sect. 7 are not shown here. The isochrones from Bertelli et al. (1994) for $Y=0.28$ and $Z=0.02$ are plotted for comparison. The numbers to the right of isochrones are the logarithms of age in years.

of the above differences transformed into reddenings and then deriving the residuals from this map for each star, (iv) selecting of non-members. In the last step, a star was identified as a non-member if its residual from the reddening map was larger than an assumed threshold. The rejected stars were not used in fitting the cluster main sequence in the next iteration. The iterations were terminated when no change in residuals occured.

The appearance of the reddening map depends on the assumed value of $\varepsilon$ (see Pigulski \& Kołaczkowski 1998 for the definition of this parameter) which, generally, is not known. The lower value of $\varepsilon$ results in a more detailed extinction map and narrower dereddened main sequence, a larger value gives the opposite effect. Fortunately, the selection of non-members is almost independent of the choice of $\varepsilon$. The main parameter which determines how many stars will be selected as non-members is the threshold adopted in step (iv) of the dereddening procedure. We have assumed the threshold to be equal to $0.1 \mathrm{mag}$. Despite some subjectivity in the method of selecting nonmembers, we think it is reasonable to use it, because it helps to find new Be stars. In Fig. 4 we see that some late-type non-members and Be stars with weak emission have similar values of $\alpha$. The selection we made allows us to distinguish fairly well between these two groups of stars.

\section{The mean reddening, distance and age}

Using the extinction map derived in Sect. 7, we corrected the CM diagram for the differential reddening. There is an advantage in using such a CM diagram for the derivation of cluster parameters, especially its age. The problem has already been pointed out by Phelps \& Janes (1994): a large spread in colours of stars close to the turn-off point led them to the conclusion that the possible range of age of NGC 663 is quite wide, between 12 and 25 Myr. An age of about 10 Myr was also proposed by Liu et al. (1991) and Tapia et al. (1991), while Mermilliod \& Maeder (1983) include NGC 663 in the group of clusters with an age of about 21 Myr.

We used our CM diagram corrected for differential reddening for the estimation of age, true distance modulus and the mean reddening of NGC 663. For this purpose we applied the isochrones of Bertelli et al. (1994) for $Y=0.28$ and $Z=0.02$. The results of fitting the 
isochrones are shown in Fig. 8. We finally adopt $11.6 \mathrm{mag}$ as the true distance modulus of NGC 663 (it corresponds to a distance of $2.1 \mathrm{kpc}$ ). This agrees very well with the recent determinations of the distance modulus given by others: $11.80 \mathrm{mag}$ (Moffat 1972), $11.55 \mathrm{mag}$ (van den Bergh \& de Roux 1978), $12.03 \pm 0.20 \mathrm{mag}$ (Tapia et al. 1991), $12.25 \mathrm{mag}$ (Phelps \& Janes 1994), and $11.6 \pm 0.7$ (Fabregat \& Torrejón 2000). The mean cluster colourexcess is $E(R-I)_{\mathrm{C}}=0.54 \mathrm{mag}$. This corresponds to $E(B-V) \approx 0.83 \mathrm{mag}$, in good agreement with other determinations (Johnson et al. 1961; Hoag 1965; Moffat 1972; Phelps \& Janes 1994). In our fit we assumed the ratio of total to selective absorption $A_{\mathrm{Rc}} / E(R-I)_{\mathrm{C}}=$ 3.7.

As can be seen in Fig. 8, the cluster upper main sequence is best fitted by the log (time in years) $=7.3$ and 7.4 isochrones. This results in the cluster age in the range between 20 and $25 \mathrm{Myr}$.

\section{Discussion}

The results of our $\mathrm{H} \alpha$ observations indicate that previous searches detected virtually all bright Be stars in NGC 663. As could be expected, our search resulted in new detections of $\mathrm{H} \alpha$ emission in late B-type stars only. The search was complete down to the A5-type stars in the cluster and revealed that Be stars are most populous in the range corresponding roughly to the spectral types between B0 and B3 (see Fig. 4). Although Be stars were also found among cluster members with the spectral type later than B3, their fraction amounts to only $7 \pm 3 \%$. Such a distribution of Be stars with spectral type was already known (Schild \& Romanishin 1976), but was derived from a small sample of stars and searches which were not complete. The case of NGC 663 confirms the dependence of Be star distribution as a function of spectral type, but more clusters of different ages need to be observed in order to come to a reliable conclusion as to this effect. $\mathrm{H} \alpha$ observations of other northern clusters rich in Be stars are now underway.

In terms of the $(R-I)_{\mathrm{C}}$ colour-index, Be stars in NGC 663 are, on average, 0.04 mag redder than nonemission stars which define the cluster main sequence. This additional reddening, presumably of circumstellar origin, is observed in almost all open clusters containing Be stars.

Acknowledgements. We are indebted to Prof. M. Jerzykiewicz for critical reading the manuscript. We also thank the referee, Dr. J. Zorec, for his valuable comments. This research was supported by the KBN grant No. 2 P03D 2909.

\section{References}

Abt, H. A., \& Golson, J. C. 1966, ApJ, 143, 306

Bertelli, G., Bressan, A., Chiosi, C., Fagotto, F., \& Nasi, E. 1994, A\&AS, 106, 275
Claría, J. J., \& Escosteguy, L. A. 1981, PASP, 93, 636

Cousins, A. W. J. 1976, Mem. RAS, 81, 25

Coyne, G. V., Wiśniewski, W., \& Otten, L. B. 1978, Vatican Obs. Publ., 1, 257

Dachs, J., \& Schmidt-Kaler, T. 1975, A\&AS, 21, 81

Danks, A. C., \& Dennefeld, M. 1994, PASP, 106, 382

Dolidze, M. V. 1975, Bull. Abastumani Astron. Obs., 47, 3

Fabregat, J., \& Torrejón, J. M. 2000, A\&A, 357, 451

Feinstein, A. 1974, MNRAS, 169, 171

Goderya, S. N., \& Schmidt, E. G. 1994, ApJ, 426, 159

González, G., \& González, G. 1954, Bol. Obs. Tonantzintla Tacubaya, 9, 3

Grebel, E. K. 1997, A\&A, 317, 448

Hoag, A. A. 1965, Vistas Astron., 8, 139

Hoag, A. A., Johnson, H. L., Iriarte, B., et al. 1961, Publ. U.S. Naval Obs., 17, 349

Jerzykiewicz, M., Pigulski, A., Kopacki, G., Miałkowska, A., \& Niczyporuk, S. 1996, Acta Astron., 46, 253

Johnson, H. L., \& Morgan, W. W. 1953, ApJ, 117, 313

Johnson, H. L., Hoag, A. A., Iriarte, B., Mitchell, R. I., \& Hallam, K. L. 1961, Lowell Obs. Bull., 5, 133

Kohoutek, L., \& Wehmeyer, R. 1997, Abhandlungen aus der Hamburger Sternwarte, Band XI

Landolt, A. U. 1992, AJ, 104, 340

Liu, T., Janes, K. A., \& Bania, T. M. 1991, AJ, 102, 1103

MacConnell, D. J., \& Coyne, G. V. 1983, Vatican Obs. Publ., 2,63

Maeder, A., Grebel, E. K., \& Mermilliod, J.-C. 1999, A\&A, 346,459

McCuskey, S. W., \& Houk, N. 1964, AJ, 69, 412

Mermilliod, J.-C., \& Maeder, A. 1986, A\&A, 158, 45

Merrill, P. W., \& Burwell, C. G. 1943, ApJ, 98, 153

Merrill, P. W., \& Burwell, C. G. 1949, ApJ, 110, 387

Merrill, P. W., \& Burwell, C. G. 1950, ApJ, 112, 72

Moffat, A. F. J. 1972, A\&AS, 7, 355

Moffat, A. F. J., \& Vogt, N. 1974, Veröff. des Astron. Institut der Ruhr-Universität Bochum, No. 2

Phelps, R. L., \& Janes, K. A. 1994, ApJS, 90, 31

Pigulski, A., \& Kołaczkowski, Z. 1998, MNRAS, 298, 753

Pigulski, A., \& Kopacki, G. 2000, A\&AS, 146, 465

Pigulski, A., Kołaczkowski, Z., \& Kopacki, G. 2000, Acta Astron., 50, 113

Pigulski, A., Kopacki, G., \& Kołaczkowski, Z. 2001, Acta Astron., in press

Sanduleak, N. 1979, AJ, 84, 1319

Sanduleak, N. 1990, AJ, 100, 1239

Schild, R., \& Romanishin, W. 1976, ApJ, 204, 493

Stetson, P. B. 1987, PASP, 99, 191

Stetson, P. B. 2000, PASP, 112, 925

Strauss, F. M., \& Ducati, J. R. 1981, A\&AS, 44, 337

Tapia, M., Costero, R., Echevarría, J., \& Roth, M. 1991, MNRAS, 253, 649

Tebbe, P. L. 1969, AJ, 74, 920

Torrejón, J. M., Fabregat, J., Bernabeu, G., \& Alba, S. 1997, A\&AS, 124, 329

Van den Bergh, S., \& de Roux, J. 1978, AJ, 83, 1075

Voight, H. H. 1965, H $\alpha$ Emission of Luminous Stars in the Northern Milky Way, part I (Bergedorf, Hamburg)

Wallenquist, Å. 1929, Medd. Från Astron. Obs. Upsala, 42 